\title{
The influence of pathogenic mutations in $\alpha$-synuclein on biophysical and structural characteristics of amyloid fibrils
}

Francesco S. Ruggeri ${ }^{1 *}$, Patrick Flagmeier ${ }^{1 *}$, Janet R. Kumita ${ }^{1}$, Georg Meisl, ${ }^{1}$ Dimitri Y. Chirgadze $^{2}$, Marie N. Bongiovanni ${ }^{1}$, Tuomas P.J. Knowles ${ }^{1,3 \#}$, Christopher M. Dobson ${ }^{1}$

${ }^{1}$ Centre for Misfolding Diseases, Department of Chemistry, University of Cambridge, Lensfield Road, Cambridge, CB2 1EW, United Kingdom

${ }^{2}$ Department of Biochemistry, University of Cambridge, Old Addenbrooke's Site, 80 Tennis Court Road, Cambridge CB2 1GA, United Kingdom

${ }^{3}$ Cavendish Laboratory, Department of Physics, University of Cambridge, Cambridge CB3 OHE, United Kingdom

\# To whom correspondence should be addressed tpjk2@cam.ac.uk,

"These authors contributed equally

Keywords: Neurodegenerative disorders, protein misfolding, single-molecule biophysics, Atomic force microscopy, infrared spectroscopy. 


\begin{abstract}
Proteinaceous deposits of $\alpha$-synuclein amyloid fibrils are a hallmark of human disorders including Parkinson's disease. The onset of this disease is also associated with five familial mutations of the gene encoding $\alpha$-synuclein protein. However, the mechanistic link between single point mutations and the kinetics of aggregation, biophysical properties of the resulting amyloid fibrils and an increased risk of disease is still elusive. Here, we demonstrate that the disease-associated mutations of $\alpha$-synuclein generate different amyloid fibrillar polymorphs compared to the wild type protein. Furthermore, the $\alpha$-synuclein variants forming amyloid fibrils of a comparable structure, morphology and heterogeneity show similar microscopic steps defining the aggregation kinetics. These results demonstrate that a single point mutation can significantly alter the distribution of fibrillar polymorphs in $\alpha$-synuclein, suggesting that differences in the clinical phenotypes of familial Parkinson's disease could be associated with differences in the mechanism of formation and the structural characteristics of the aggregates.
\end{abstract}




\section{INTRODUCTION}

Over the past two decades, the conversion of soluble proteins into misfolded amyloid fibrils has generated a very great deal of attention because of its link with neurological disorders such as Parkinson's disease. ${ }^{1,2}$ Furthermore, it is now recognized that the formation of amyloid deposits in organs and tissues is associated with more than fifty human diseases ${ }^{1}$ and the existence of different strains and structural conformations of the protein aggregates has been related to variations in pathological phenotypes and to the onset of different pathologies. ${ }^{3,4}$ The toxicity of protein aggregates associated in particular with neurodegenerative diseases is widely believed to be a result of their ability to disrupt membranes, ${ }^{5}$ interact with receptor proteins, ${ }^{6}$ sequester cellular components ${ }^{7}$ or to generate highly reactive damaging species ${ }^{8}$. The process of amyloid formation has, however, not only been related to pathology, as functional amyloid structures have also been found, for example in the context of the reversible formation of structures for the storage of hormones. ${ }^{9}$ Defining the underlying mechanisms of fibril formation and identifying the structures present on the pathways through which they are formed, are therefore important factors in the development of potential treatments for protein misfolding diseases. ${ }^{10}$ In addition, an enhanced understanding of the factors affecting the formation of amyloid structures provides opportunities for the development of novel materials and devices. $^{11-14}$

The overall architecture of amyloid fibrils has been found to be independent of the protein sequence, and of their native state structures. ${ }^{1}$ Indeed, amyloid fibrils formed from different proteins have often been found to possess strikingly similar structural characteristics, including long, unbranched and twisted morphologies and diameters in the range of a few nanometers with lengths of the order of micrometers. ${ }^{15,16} \mathrm{~A}$ universal characteristic of amyloid fibrils is a cross- $\beta$ sheet structure in the individual protofilaments that together form the final structures. ${ }^{1}$ Despite the high degree of overall structural similarity of amyloid fibrils formed from different proteins, the details of individual structures are dependent on the sequences involved and the conditions under which they are formed, and a high degree of polymorphism and heterogeneity is frequently observed. ${ }^{17-20}$ Moreover, single amino acid substitutions or small changes in $\mathrm{pH}$ have been reported to result in a change in a variety of distinctive structural features of amyloid fibrils and their precursors. ${ }^{21-24}$

Misfolding diseases are in many cases sporadic, but they can also be promoted by mutations in the proteins concerned. In the case of Parkinson's disease (PD), sporadic onset of pathology is closely associated with the misfolding and aggregation of the protein $\alpha$-synuclein, which has been found to be a major constituent of deposits in brains of individuals suffering 
from PD. ${ }^{25}$ This protein is composed of 140 amino acid residues and has an intrinsically disordered structure in solution. It has been found to be expressed in nerve cells where it is primarily localized at synaptic terminals and the protein appears to play a role in synaptic plasticity. ${ }^{26,27}$ Its sequence can be subdivided into an amphipathic N-terminal region that interacts with negatively charged surfaces, such as lipid bilayers, and induces $\alpha$-helical secondary structure, a central region referred to as the non-amyloid $\beta$-component (NAC) that has a high aggregation propensity, and an acidic C-terminal domain that is typically highly disordered. A series of mis-sense mutations in the SCNA gene encoding $\alpha$-synuclein have been found to be associated with familial forms of the disease. These mutations result in the replacement of a single amino acid residue at different positions in the sequence, namely $\mathrm{A} 30 \mathrm{P},{ }^{28} \mathrm{E} 46 \mathrm{~K},{ }^{29} \mathrm{H} 50 \mathrm{Q},{ }^{30} \mathrm{G}^{2} \mathrm{D}^{31}$ and A53T. ${ }^{27,} 32$ The mutations have been reported to alter the rate of $\alpha$-synuclein aggregation in vitro, changing to varying extents one or more microscopic steps in the aggregation mechanism. ${ }^{21}$ It has also been suggested that the mutations may influence the morphology of the resulting aggregation products, ${ }^{21}$ although the extent of such changes remains to be explored. Furthermore, the link between the aggregation kinetics and the aggregate structures is of considerable interest as it is likely that this relationship is an important factor contributing to the onset of disease.

The properties of amyloid fibrils in vitro have been investigated by a range of bulk biophysical techniques, such as X-ray diffraction patterns and circular dichroism. These methods provide averaged measurements, which are not generally able to define the complex heterogeneous mixture of amyloid aggregates. In order to gain insight into the individual structural species present under given solution conditions, techniques such atomic force microscopy (AFM) can be applied to study individual fibrils. ${ }^{33,}{ }^{34}$ In particular, AFM has been demonstrated to be a powerful technique able to provide high resolution information at the single molecule level about the 3-D morphological properties of individual amyloid fibrils, such as their cross-sectional dimensions and periodicity. ${ }^{10,35-37}$

In the present work, we set out to characterize systematically the influence of the disease-associated mutations on the structural characteristics of amyloid fibrils formed by $\alpha$ synuclein. To achieve this objective, it was necessary to generate the fibrils from the monomeric forms of all variants under identical and highly controlled conditions such that the differences in the fibril populations can be attributed to the intrinsic differences in the sequence of the protein rather than to variations in the growth process. We first investigated the biophysical properties of the final products of the aggregation reaction by means of a range of conventional biophysical techniques, including X-ray diffraction, circular dichroism and 
infrared spectroscopies. We then identified morphological differences in the fibril samples using TEM imaging. Then, we used 3-D high-resolution mapping by AFM, in combination with an unsupervised k-means machine learning clustering, to define the polymorphism and heterogeneity of the ensemble of structures formed by the different protein variants with subnanometer resolution. By characterizing the fibrils of the mutational variants in this way, we identified distinct morphological categories that have enabled us to observe a correlation between the differences in the various mechanistic steps involved in amyloid fibril formation, observed in previous studies, ${ }^{21}$ and the structural nature of the populations of the fibrillar aggregates formed by the different variants of $\alpha$-synuclein. 


\section{RESULTS}

\section{Preparation of amyloid fibrils from $\alpha$-synuclein variants}

In order to perform a systematic characterization of the influence of the pathogenic single-point mutations on the morphology of the mature fibrils, external factors such as the $\mathrm{pH}$ value, salt and temperature had to be excluded. Therefore, it was necessary to prepare fibrils from the WT protein, and from the mutational variants under identical and specifically designed solution conditions (Fig. 1a,b). We chose neutral $\mathrm{pH}$ and quiescent incubation conditions in order to minimize the influence of surfaces, ${ }^{23}, 38,39$ variation in solution conditions ${ }^{21,22}$ and fibril fragmentation. ${ }^{40} \mathrm{We}$ incubated the monomeric proteins in the presence of pre-formed fibrils of their respective variants (SI Appendix Text, Fig. S1).

\section{The fibrils formed by all variants show classical amyloid characteristics}

The initial analysis of the resulting aggregates was carried out with the fluorescent dyes 8anilinonaphthalene-1-sulfonic acid (ANS) ${ }^{41}$ and Nile $\operatorname{Red}^{42}$. The results revealed shifts of the emission wavelengths characteristic of ordered amyloid structures for the aggregates of all the variants (SI Appendix Fig. S1).

The secondary structure of monomeric $\alpha$-synuclein, observed by methods such as circular dichroism (CD) and Fourier transform infrared (FTIR) spectroscopies, is essentially random coil in nature (SI Appendix Fig. S1), but upon the formation of fibrils a dramatic change in the spectra occurs indicating the formation of a high content of $\beta$-sheet structure. ${ }^{15}$, ${ }^{43}$ The fibrils formed by the G51D variant displayed two distinct minima of the CD spectra at $208 \mathrm{~nm}$ and $222 \mathrm{~nm}$, indicative of a higher level of $\alpha$-helical structure content compared with the fibrils formed from the other variants (SI Appendix, Table S1).

FTIR spectroscopy was used for a more detailed analysis of the $\beta$-sheet structure. We recorded spectra of the monomeric and fibrillar states of all the variants (Fig. 1c,d), and analysed the amide I band in detail. ${ }^{44-46}$ The FTIR spectra of all the monomeric variants were found to be indistinguishable showing that the monomeric state of each variant adopts a natively unfolded structure (Fig. 1c), ${ }^{47}$ whereas the spectra of the fibrillar state of all variants showed a substantial enrichment in $\beta$-sheet structure for the fibrils with differences in the region of the amide I band revealing distinct structural features (Figure 1e,f and SI Appendix Fig. S2, Tables S2,3).

We first quantified by difference spectroscopy the amount of total structural change of the amide band I introduced by each mutational variant, respect to the structure of WT fibrillar aggregates (Fig. 1g and SI Appendix Fig. S3). The analysis demonstrates that A30P and A53T 
mutational variants have much more similar structure than E46K, H50Q and G51D mutations. In order to quantify further the relative content of the different types of secondary structure in the fibrils formed by the variants, we calculated the second derivative of the amide I band of each FTIR spectrum ${ }^{43,48}$ (SI Appendix Fig. S3). The $\alpha$-helical content of the E46K variant was lower, and that of the G51D variant higher compared to the other variants. In addition, we examined the ratio between the relative content of parallel and antiparallel $\beta$-sheet to the content of $\alpha$-helical and $\beta$-turn structure (Fig. 1h and SI Appendix Fig. S3). While the WT, A30P and A53T variants had similar ratios of these structural features, the remaining mutational variants (E46K, H50Q, and G51D) were significantly different, demonstrating a change in the details of the structural organization. Overall, the analysis of the FTIR data demonstrates that the disease related single point mutations of $\alpha$-synuclein form two structurally different groups of fibrillar aggregates. The first group of mutations results in fibrillar aggregates structurally similar to WT fibrils (A30 and A53T), while the second group of point mutations (E46K, H50Q, G51D) results is significant differences in the structure of the fibrillar aggregates compared to the WT ones (Fig. 1g-h).

We next examined the fibrils by X-ray diffraction, to probe the presence of the ordered cross- $\beta$ structure characteristic of the amyloid architecture. All variants showed the characteristic anisotropic diffraction pattern with reflections at $4.7 \AA$ (inter-strand spacing) and $10 \AA$ A (inter-sheet spacing) (Fig. 1i). ${ }^{49}$ Next, we analysed the fibrils using TEM and observed the presence of a very significant degree of heterogeneity, showing the presence of both straight, rod-like fibrils and twisted rope-like species in each case (SI Appendix Fig. S3).

\section{Analysis of individual fibrils reveals morphological differences in the fibrils formed by the variants}

We next acquired high-resolution and phase controlled 3-D maps of the fibrils from the different variants by using AFM (Fig. 2). ${ }^{50}$ Our aim was to investigate and quantify the level of polymorphism in the fibrillar samples of each variant, performing a statistical analysis of the cross-sectional dimensions and packing of the individual fibrils (Fig. 3 and SI Appendix Fig. S4-5). In particular, we aimed at investigating the structural properties of the aggregates in comparison with the previously described influence on the overall kinetics and microscopic steps of amyloid formation (Fig. 4a,b). We found for all fibrillar variants the co-existence of polymorphic rod-like and twisted fibrils, with a defined periodicity and fluctuations of the height along the fibril length. ${ }^{20,37}$

We first performed a statistical analysis of the cross-sectional height, which correspond to the diameters of structures with cylindrical symmetry, of the populations of the non-periodic 
fibrils (Fig. 3b and SI Appendix Fig. S4). The WT, A30P, A53T protein showed two populations with rod-like morphologies. By contrast, for fibrils formed from the E46K and H50Q variants, we found only one group of rod-like fibrils with a significantly smaller diameter than WT. In the case of the G51D variant, however, we observed the formation of effectively a single population of twisted fibrils in the AFM maps. The analysis showed that the rod-like fibrils of the mutant variants had smaller cross-sectional diameter and a reduced number of rod-like polymorphs than WT fibrils (Fig. 3b and SI Appendix Table S4). These results are in excellent agreement with previous studies indicating by atomistic models that $\alpha$-synuclein mutants should disfavour the formation of rod-like amyloid fibrils over twisted fibrils

Then, we analysed the twisted fibrillar species to study their periodicity and internal packing (Fig. 3c-d). The pitch along the maximum height profile was defined as the distance between two maximal heights of a fibril. Furthermore, we analysed the average difference between the maximum and minimum height of a fibril (max-min height difference) (Fig. 3cd). Twisted fibrils were found for all the variants. The existence of significantly different twisted polymorphs within each variant of $\alpha$-synuclein was demonstrated by an unsupervised machine learning $k$-means clustering analysis performed in the morphological space of height, pitch and min-max height of the fibrils (Fig. 3 and SI Appendix Text). The mutational variants showed a smaller average pitch and height compared to the polymorphs present in the samples of the WT protein (Fig. 3 and SI Appendix Table S4). The pitch and the cross-sectional diameter of the fibrils formed by the A30P and A53T variants were close to those of the WT protein, while, the fibrils formed by three of the mutational variants (E46K, H50Q, and G51D) were observed to possess a significantly smaller periodicity and reduced cross-sectional diameter (Fig. 3 and SI Appendix Table S4) compared to the WT protein.

Both rod-like and twisted polymorphs of the variants showed a smaller cross-sectional height than the WT protein (Fig. 3 and SI Appendix Table S4). The diameter of a fibril is linked to the number of protofilaments assembled together in a fibril and the pitch and the maxmin height difference are related to their geometrical packing and structural organization. ${ }^{37,51,}$ ${ }^{52}$ In the case of twisted and compact helical ribbon cross-sectional arrangements of fibrils, the maximum cross sectional diameter scales with the number of protofilaments based on geometrical constraints. For a twisted arrangement, the radius of a fibril $(d)$ is proportional to the radius $\left(d_{0}\right)$ of the elementary protofilament as $d \approx n d_{0}$, while for a helical arrangement the correlation has been described as $d \approx n^{1 / 2} d_{0}{ }^{53,54}$ The finding of a smaller diameter for the fibrils formed from the mutational variants, in particular, the E46K, H50Q and G51D fibrils, therefore 
demonstrates that they consist of a smaller number of protofilaments compared to the fibrils formed by the WT protein.

We can summarize our results to conclude that single point mutations have a profound effect on the nature of the structure of fibrils, by considering the data from the perspective of a three-dimensional morphological space, with the parameters of height, pitch and min-max height variation of the average fibril populations (Fig. 4b). In this morphological space, we observed that the fibrils of the WT protein and the A30P and A53T variants have similar localization and morphological properties (SI Appendix Table S4), but that the fibrils formed by the H50Q, E46K and G51D variants are spatially separated and hence morphologically different. The grouping of the two morphologically distinct class of mutations in the 3-D space of height, pitch and min-max height was further demonstrated by the unsupervised machine learning k-means clustering analysis (SI Appendix Text, Fig. S6). Remarkably, the morphological properties of the rod-like and twisted fibrils formed by the variants sub-grouped similarly to the previously found sub-grouping in the microscopic steps of the kinetics of aggregation (circles in Fig. 4a,b). ${ }^{21}$

The projection of the 3-D morphological space on the 2-D plane of height vs. min-max height difference shows fundamental morphological differences between the variants (SI Appendix Text, Fig. S7). The E46K, H50Q and G51D variants can form fibrils in either a helical or a twisted ribbon arrangement, where the min-max height profile accounts for approximately $20 \%$ of the maximal height. Whereas the WT, A30P and A53T variants form fibrils where the min-max height profile accounts for approximately $20 \%$ of the maximal height, thus suggesting a helical ribbon arrangement with more compact cross-sectional packing. ${ }^{53,55}$

Finally, the projection of the 2-D morphological space on the height vs. pitch plane shows that the fibrillar pitch increases as a function of the average height of the fibrils independently from the single point mutations (Fig. 4c). A general correlation between the pitch $p$ and the cross-sectional diameter $d$ of amyloid fibrils has previously been demonstrated by Knowles et $a l^{54}$ and it can be written as: $p=\frac{p_{0}}{2} \cdot\left(1+\sqrt{1-\frac{4 a_{T}^{b}}{k_{T}^{s}} * d^{2}}\right)$, where $p_{0}$ is the minimal pitch for the system, $k_{T}^{s}$ is the inter-strand torsional spring constant and $k_{T}^{b}=a_{T}^{b} \cdot d^{2}$ is the bulk torsional spring constant of the fibril. ${ }^{53,54} \mathrm{We}$ used this relationship to fit the average height versus the pitch of our results and previous literature data of amyloid fibrils formed by several different peptides and by $\alpha$-synuclein (red line in Fig. 4c). We extrapolated from the fit a value of $\frac{a_{T}^{b}}{k_{T}^{s}}=0.04 \pm 0.01 \mathrm{~nm}^{-2}$ and $p_{0}=60 \pm 15 \mathrm{~nm}$. Remarkably, though the 
relationship does not account for differences between different proteins or chemical modification induced by point mutations, the extracted parameters correspond well with previous reports in the literature. ${ }^{18,56-59}$ Remarkably, the structural polymorphs obtained from human WT $\alpha$-synuclein belong to the same morphological space of the amyloid fibril obtained in vitro. ${ }^{18}$ The ability of this general relationship to fit the data extrapolating significant physical constants (Fig. 4) supports the existence of a general model of a hierarchical assembly mechanism for $\alpha$-synuclein amyloid fibril formation, where prefibrillar species intertwine to form protofibrils which then intertwine further to form mature amyloid fibrils. ${ }^{10,60,61}$ 


\section{DISCUSSION}

We have demonstrated by means of a range of bulk biophysical techniques that aggregates formed by WT $\alpha$-synuclein, and all the mutational variants (A30P, E46K, H50Q, G51D, and A53T) known to be associated with familial forms of Parkinson's disease display the classic features of amyloid fibrils (i.e. a high $\beta$-sheet content, a cross $\beta$-structure, and the ability to bind dyes such as ThT). By means of CD and FTIR spectroscopies, we have shown that the monomeric forms of all the variants have natively unfolded structures in aqueous solution (Fig.1c-e). By contrast, the secondary structural content of the fibrillar state of the WT protein is similar to A30P and A53T fibrils, while it differs from that of the other disease-associated variants, E46K, H50Q and G51D (Fig.1f-h).

The imaging of the aggregates with TEM and AFM shows the presence of long polymorphic fibrillar structures in the case of all the variants. The statistical analysis of the high-resolution 3-D AFM maps is consistent with the results of the bulk characterization of the fibrils, but enables the study of their heterogeneity at the single aggregate scale with subnanometer resolution. We then exploited unsupervised machine learning clustering to differentiate accurately the fibrillar polymorphs formed by the WT and mutant variants. When the structural features are compared in three-dimensional morphological space, the fibrils formed by the mutational variants separate spatially from the fibrils formed by the WT protein (Fig. 4b). In particular, G51D, E46K and H50Q form a group (indicated by a green circle, Fig. 4b,c) distinct from the WT protein and the A30P and A53T variants (indicated by a red circle, Fig. 4b,c). The fibrils formed by mutational variants, and in particular E46K, H50Q, and G51D, were observed to possess a significantly smaller periodicity and a reduced cross-sectional diameter compared to the WT protein, indicating that the fibrils consist of a smaller number of constituent protofilaments. In the cases of one population of the E46K, H50Q and G51D variants, we found also a larger variation of min-max-height with respect to the average height of the fibrils; indicating that these polymorphs have a different cross-sectional packing of their fibril core when compared to the WT protein (SI Appendix Fig. S7). Furthermore, we found that the pitch as a function of periodicity follows the previously found general relationship governing the phase transition between twisted and crystal fibrils. ${ }^{56-59}$ These results are in good agreement with previous experimental data reported in the literature (yellow shapes in Fig. 4c) and supporting the existence of a general model of hierarchical assembly for $\alpha$-synuclein fibril formation, independently of the different mutations.

In light of the morphological and structural changes induced by the disease-associated mutations, we compared the influence of the mutations on the structural characteristics 
described here with previous descriptions of their influence on the kinetics of amyloid formation (Fig. 4a,b). ${ }^{21}$ All of these mutations in $\alpha$-synuclein have been found to influence the overall aggregation kinetics. $^{21,29,62}$ The E46K, H50Q, and A53T variants were reported to aggregate faster, ${ }^{30,63-65}$ the G51D variant slower, ${ }^{62}$ and the A30P variant with comparable, ${ }^{66}$ slower or faster rates, ${ }^{64}$ compared to the WT protein under varying conditions of agitation. The different mutations were found to alter the rates of one or more of the microscopic events in the aggregation mechanism ${ }^{21}$ under very different conditions, though all in quiescent conditions, namely to initiate aggregation in the presence of vesicles and to promote aggregate proliferation under acidic conditions. Strikingly, as proved by an unsupervised clustering machine learning algorithm (SI Appendix Text), we found an identical sub-grouping of the variants in the structural data as in the reported kinetic data. The A30P and A53T variants were found to aggregate in a similar way to the WT protein under the acidic conditions (red circle, Fig. 4a), whereas aggregation of the H50Q and G51D variants was not detectable under the acidic conditions (green circle, Fig. 4a). The rate of aggregation of the E46K variant was on the one hand found to be comparable to that of the WT, A30P and A53T variants in the presence of vesicles, but on the other hand to be comparable to the H50Q and G51D variants under acidic conditions. ${ }^{21}$

In summary, the observations of the behaviour and sub-grouping of the different mutational variants from a kinetic perspective can be correlated with the sub-grouping of the structural and morphological features of the resulting fibrils, as observed by CD, FTIR, TEM and AFM (Fig. 2, Fig. 3 and Fig. 4a-c). Taken together, these results suggest that differences in the morphological features of the fibrils are related to the mechanistic differences associated with their formation (SI Appendix Fig. S8). In Fig. 4d, it is represented the three-dimensional diagram of the morphological height, the rate of lipid induced aggregation and of secondary structural differences of amyloid fibrils. The A53T and A30P variants show similar kinetic constants, structure and morphology when compared to the WT protein, while the E46K, H50Q and G51D variants show large differences in secondary structure, morphology and in the microscopic steps in the aggregation mechanism relative to the WT protein. Finally, the results indicate that the existence of a general correlation between the mechanism of amyloid formation, the morphology and the structure of the final fibrillar products of the aggregation reaction). 


\section{CONCLUSIONS}

Individuals with a range of mutations in the SCNA gene that encodes the genetic information for the expression of $\alpha$-synuclein have been reported to be more prone to developing Parkinson's disease than those carrying the wild type sequence, often with an earlier age of onset. ${ }^{67}$ Details of reported cases of familial Parkinson's disease have, however, often been contradictory, some indicating phenotypic differences and some others suggesting that the characteristics of the familial diseases are very similar to those of the sporadic cases involving the WT protein. ${ }^{68}$ The present results reveal that the disease-associated mutations, involving the substitution of a single amino acid residue in the 140 residue sequence of $\alpha$ synuclein, can alter the populations and morphologies of the amyloid fibrils formed by the different variants. We have in addition observed for the disease-associated mutational variants of $\alpha$-synuclein that differences in the structural features of the amyloid fibrils are associated with differences in vitro mechanism of their formation. The existence of different protein strains and structural variants in fibril populations has been related to variations in pathological phenotypes, not just in the case of $\alpha$-synuclein, but also of the amyloid $\beta$-peptide associated with Alzheimer's disease, ${ }^{69}$ and the prion protein linked to Creutzfeldt-Jakob disease. The observations here indicate that mutations in the gene encoding $\alpha$-synuclein can alter significantly the nature of the fibrillar structures and suggest that differences in the clinical phenotypes of familial Parkinson's disease could be associated with differences in the structural characteristics of the aggregates as well as with the kinetics of their formation. Understanding how point mutations influences amyloid fibril morphology and the mechanism

of protein aggregation in this, and other fibril forming systems, should contribute significantly to the development of strategies to combat protein misfolding disorders. 


\section{MATERIALS AND METHODS}

Detailed information on the experimental methods can be found in the SI Appendix. Recombinant monomeric $\alpha$-synuclein or its mutational variant was incubated at a concentration of $100 \mu \mathrm{M}$ in the presence of sonicated fibrils of the respective variant at a concentration of 5 $\mu \mathrm{M}$, without and with $50 \mu \mathrm{M}$ ThT at $37^{\circ} \mathrm{C}$ under quiescent conditions. X-ray diffraction patterns were recorded on a Microstar microfocus rotating anode X-ray generator equipped with a Platinum $135 \mathrm{CCD}$ detector. Attenuated total reflection infrared spectroscopy was performed using a Bruker Vertex 70 spectrometer equipped with a diamond ATR element. Atomic Force Microscopy was performed on positively functionalized mica substrates by means of a NX10 (Park Systems) operating in tapping mode and equipped with a silicon tip $\left(\mathrm{PPP}-\mathrm{NCHR}, 5 \mathrm{Nm}^{-1}\right.$ ) with a nominal radius $<10 \mathrm{~nm}$.

\section{DATA AVAILABILITY}

All data discussed in the paper will be made available to readers.

\section{ACKNOWLEDGEMENTS}

This study was supported by the Boehringer Ingelheim Fonds (P.F.), the German National Merit Foundation (P.F.), the Swiss National Science Foundation and Darwin College (F.S.R.), the UK Biotechnology and Biochemical Sciences Research Council (C.M.D.); the Wellcome Trust (C.M.D. and T.P.J.K.); the Frances and Augustus Newman Foundation (T.P.J.K.) and the Cambridge Centre for Misfolding Diseases (P.F., F.S.R., J.R.K., M.N.B., T.P.J.K. and C.M.D.). 


\section{FIGURES}
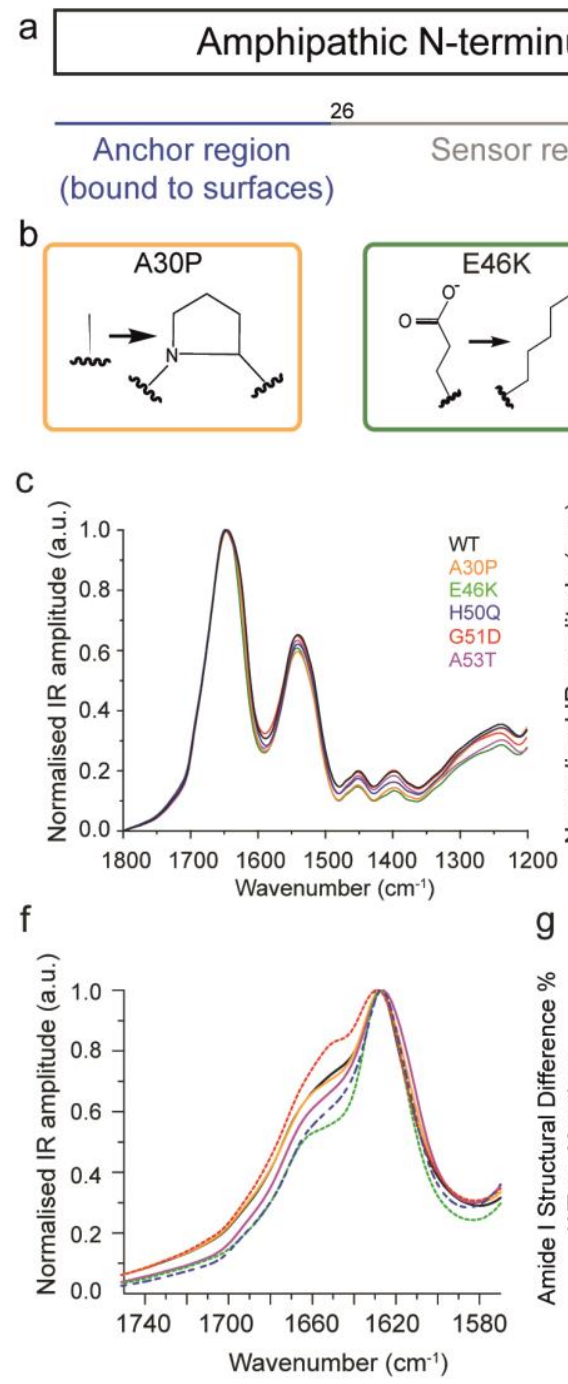

i WT
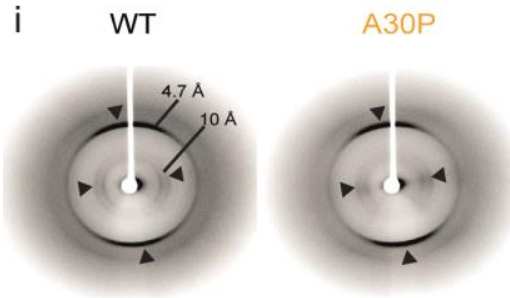
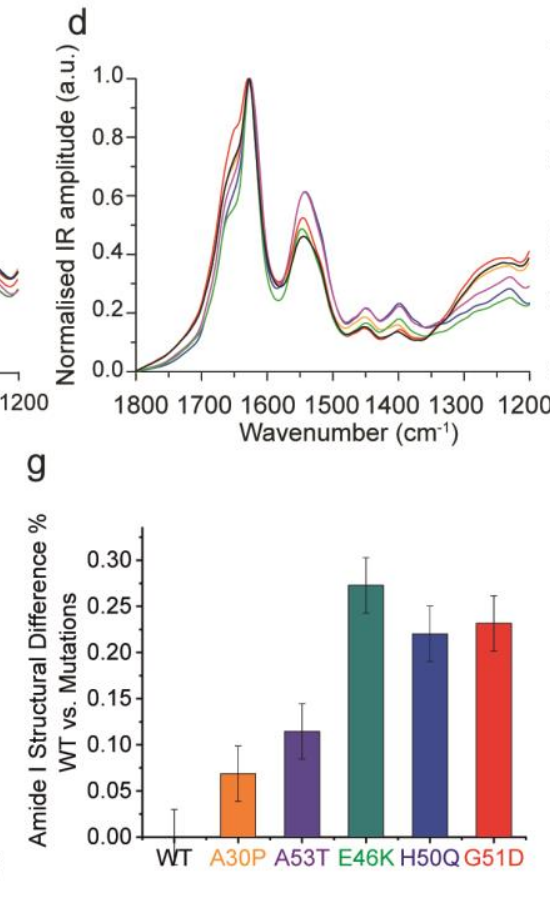

$\mathrm{h}$
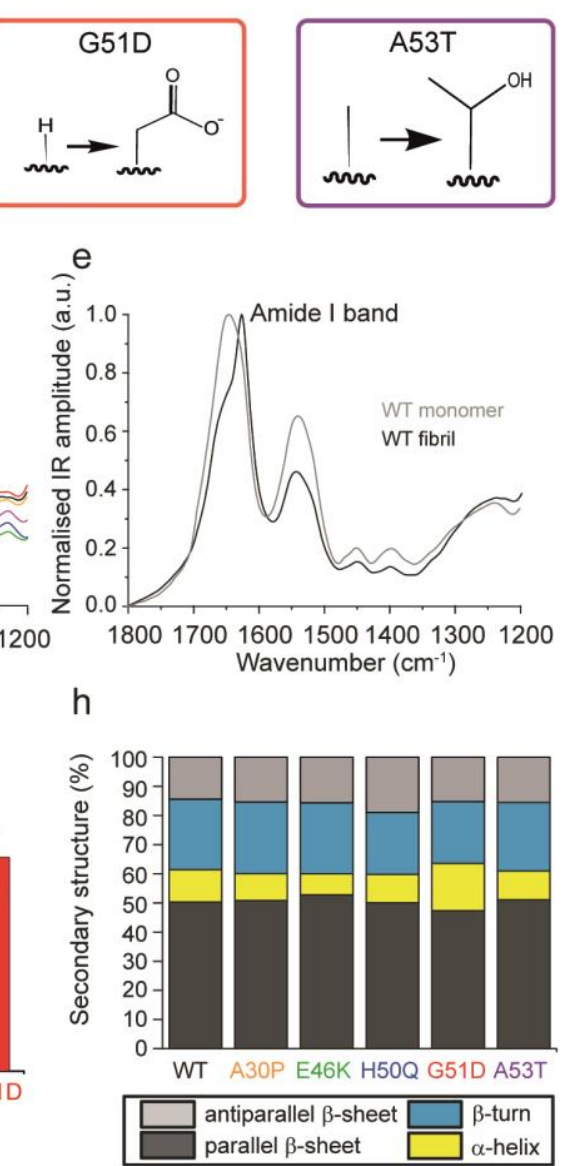

E46K

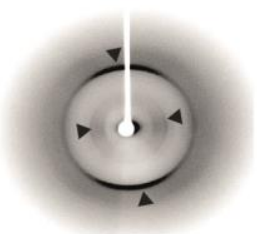

H50Q

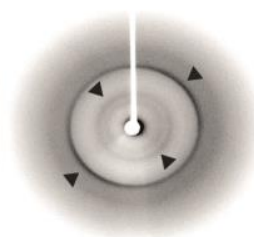

G51D

A53T

Figure 1: Amino acid sequence, secondary structure and $c$ ross- $\beta$ diffraction patterns of $\alpha$ synuclein and its mutational variants associated with familial Parkinson's disease. (a) The structure of $\alpha$-synuclein can be divided into three regions: the amphipathic N-terminal domain (1-60), the non-amyloid $\beta$-component domain (NAC, 61-94) and the acidic C-terminal domain (95-140). (b) The mis-sense mutations in the SCNA gene encoding $\alpha$-synuclein result in the replacement of singleamino acid residues that change the chemical composition of the protein. Normalized FTIR spectra of the (c) monomeric and the (d) amyloid fibrils of WT protein and the mutational variants. (e) Spectra of the WT protein in its monomeric (light grey) and fibrillar states (dark grey). (f) Normalised spectra in the amide I band. (g) Amide I structural difference of each mutational variant respect to WT. (h) Relative secondary structure content for $\alpha$-synuclein and its mutational variants based on the second derivative of the FTIR spectra. (i) Characteristic anisotropic cross- $\beta$ fibre diffraction pattern of amyloid fibrils formed by WT $\alpha$-synuclein and its mutational variants. The anisotropy of the diffraction patterns at approximately $4.7 \AA$ (inter-strand spacing) and $10 \AA$ (inter-sheet spacing) are indicated by the arrow heads. 

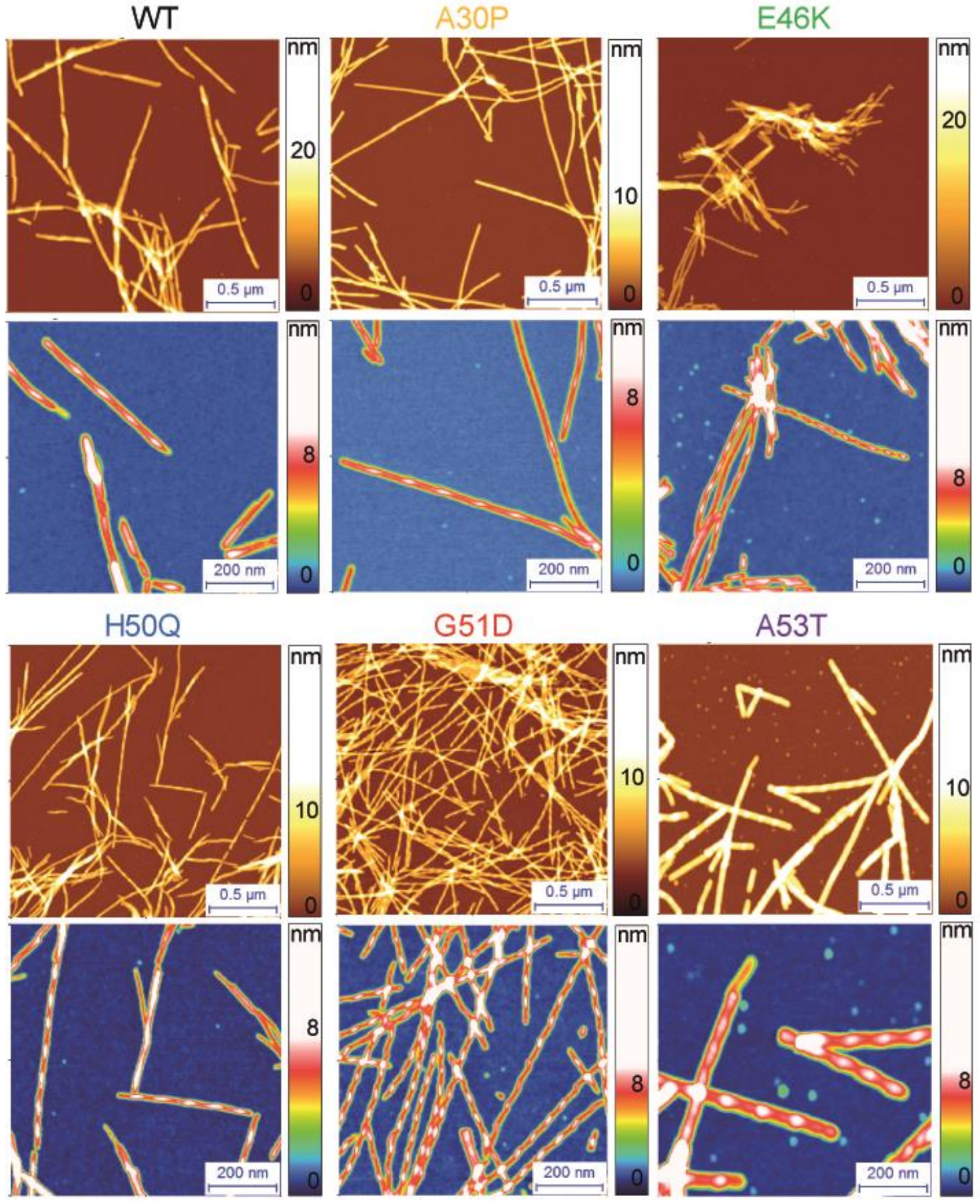

Figure 2: High-resolution AFM images of WT $\alpha$-synuclein and its disease-associated mutational variants. For each sample a series of three-dimensional AFM maps were acquired under conditions of phase change monitoring ${ }^{50}$ in order to compare the morphology of the fibrils quantitatively. 
a
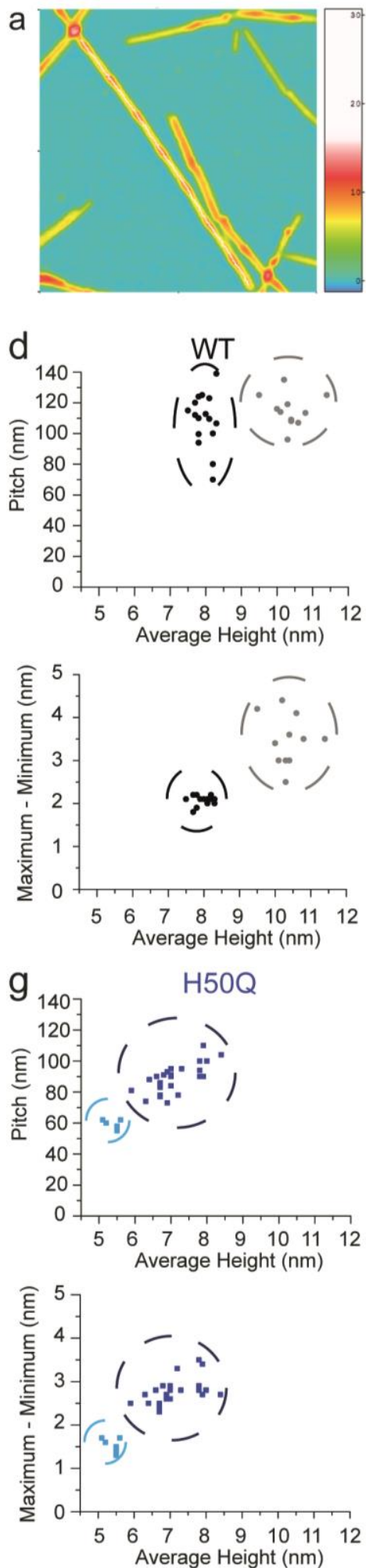
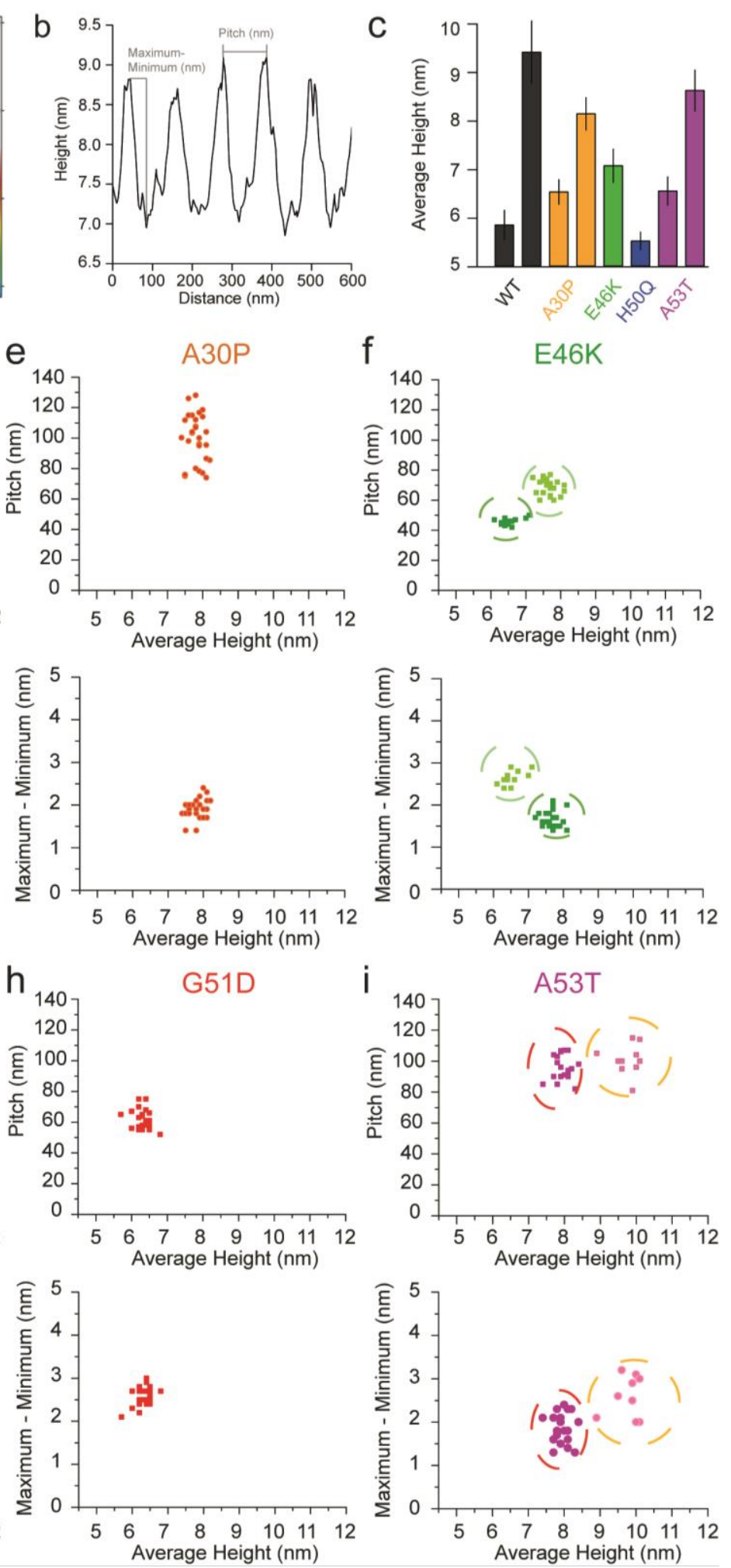

Figure 3: Polymorphism of the fibrils formed by WT $\alpha$-synuclein and the mutational variants associated with familial Parkinson's disease. (a) AFM image of fibrils formed by WT $\alpha$-synuclein. (b) Analysis of morphological features at the single fibril level. (c) The average height of non-periodic fibril populations for the WT protein and the A30P (orange), E46K (green), H50Q (blue) and A53T (purple) variants. (d-i) Representation of the periodic fibrils of each variant in the height vs. pitch and height vs. max-min planes. Each point in the graph represent the morphology parameters of a single fibril. Unsupervised machine learning clustering analysis determined that WT, E46K, H50Q and A53T form 2 different polymorphs, while G51D and A30P fibrils did not form clearly different polymorphs. The dashed circles are used as guidelines to visualise the points assigned by the algorithm to each cluster. For each variant, we analysed the 3-D morphology of at least $n>45$ fibrils. 

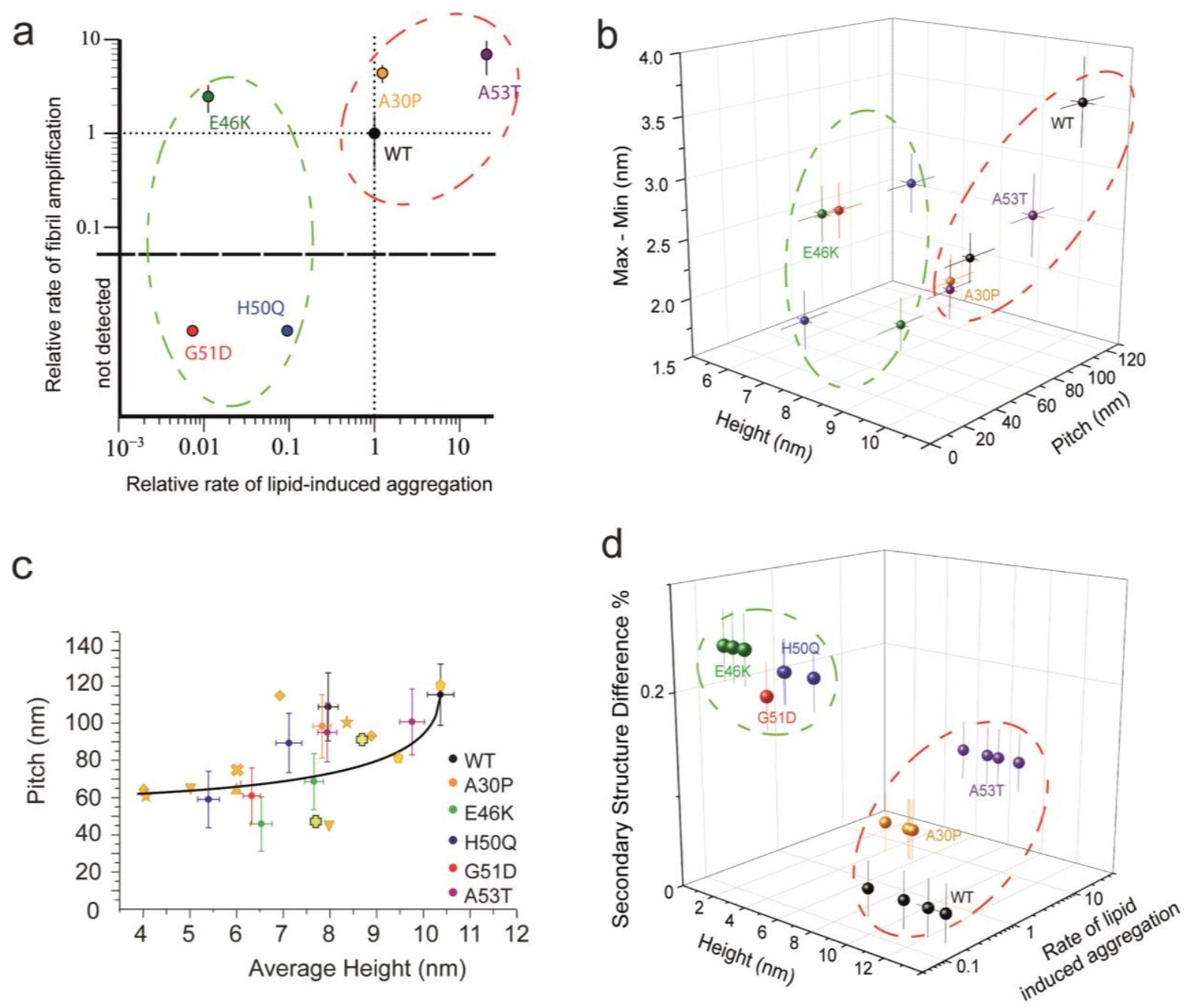

Figure 4: Clustering of structural alterations and changes in the microscopic aggregation steps for the disease-associated mutations in $\alpha$-synuclein. (a) Previously found microscopic rates of fibril amplification and lipid-induced aggregation (Reproduced from ${ }^{21}$ ) show that WT, A30P and A53T cluster with similar kinetics rates (red circle), G51D and H50Q cluster separately (green circle), while E46K has intermediate properties. (b) Three-dimensional diagram of the morphological features of amyloid fibrils. The fibrils formed by the WT protein and the A30P and A53T variants share morphological space (red circle), whereas the fibrils formed by the H50Q, E46K and the G51D variants occupy different regions of morphological space (green circle). The ellipses have been added to emphasize the inter-mutant sub-grouping of each polymorph obtained by a $k$-means clustering analysis. (c) The pitch plotted versus the average height of the fibrils. The black line is a fit of the data from the present manuscript (circles) and data from previous studies (yellow shapes) using the previously demonstrated general relationship between the twist angle and the diameter of the fibrils. ${ }^{18,19,56-59,70}$ (d) Three-dimensional diagram of the morphological height, the rate of lipid induced aggregation and of secondary structural differences of amyloid fibrils (Fig. 1g). The fibrils formed by the WT protein and the A30P and A53T variants share (red circle) group separately than the fibrils formed by the H50Q, E46K and the G51D variants (green circle). 


\section{REFERENCES}

1. Chiti, F.; Dobson, C. M. Protein Misfolding, Amyloid Formation, and Human Disease: A Summary of Progress Over the Last Decade. Annu Rev Biochem 2017, 86, 27-68.

2. Aguzzi, A.; O'Connor, T. Protein aggregation diseases: pathogenicity and therapeutic perspectives. Nat Rev Drug Discov 2010, 9, 237-48.

3. Guo, J. L.; Covell, D. J.; Daniels, J. P.; Iba, M.; Stieber, A.; Zhang, B.; Riddle, D. M.; Kwong, L. K.; Xu, Y.; Trojanowski, J. Q.; Lee, V. M. Distinct alpha-synuclein strains differentially promote tau inclusions in neurons. Cell 2013, 154, 103-17.

4. Peelaerts, W.; Bousset, L.; Van der Perren, A.; Moskalyuk, A.; Pulizzi, R.; Giugliano, M.; Van den Haute, C.; Melki, R.; Baekelandt, V. alpha-Synuclein strains cause distinct synucleinopathies after local and systemic administration. Nature 2015, 522, 340-4.

5. Flagmeier, P.; De, S.; Wirthensohn, D. C.; Lee, S. F.; Vincke, C.; Muyldermans, S.; Knowles, T. P. J.; Gandhi, S.; Dobson, C. M.; Klenerman, D. Ultrasensitive Measurement of Ca2+ Influx into Lipid Vesicles Induced by Protein Aggregates. Angewandte Chemie International Edition 2017, 56, 7750-7754.

6. Haass, C.; Selkoe, D. J. Soluble protein oligomers in neurodegeneration: lessons from the Alzheimer's amyloid beta-peptide. Nature Reviews Molecular Cell Biology 2007, 8, 101-112.

7. Woerner, A. C.; Frottin, F.; Hornburg, D.; Feng, L. R.; Meissner, F.; Patra, M.; Tatzelt, J.; Mann, M.; Winklhofer, K. F.; Hartl, F. U.; Hipp, M. S. Cytoplasmic protein aggregates interfere with nucleocytoplasmic transport of protein and RNA. Science 2016, 351, 173-6.

8. Soto, C. Unfolding the role of protein misfolding in neurodegenerative diseases. Nature Reviews Neuroscience 2003, 4, 49-60.

9. Maji, S. K.; Perrin, M. H.; Sawaya, M. R.; Jessberger, S.; Vadodaria, K.; Rissman, R. A.; Singru, P. S.; Nilsson, K. P.; Simon, R.; Schubert, D.; Eisenberg, D.; Rivier, J.; Sawchenko, P.; Vale, W.; Riek, R. Functional amyloids as natural storage of peptide hormones in pituitary secretory granules. Science 2009, 325, 328-32.

10. Ruggeri, F. S.; Benedetti, F.; Knowles, T. P. J.; Lashuel, H. A.; Sekatskii, S.; Dietler, G. Identification and nanomechanical characterization of the fundamental single-strand protofilaments of amyloid alpha-synuclein fibrils. Proc Natl Acad Sci U S A 2018, 115, 7230-7235.

11. Schilling, C.; Mack, T.; Lickfett, S.; Sieste, S.; Ruggeri, F. S.; Sneideris, T.; Dutta, A.; Bereau, T.; Naraghi, R.; Sinske, D.; Knowles, T. P. J.; Synatschke, C. V.; Weil, T.; Knöll, B. SequenceOptimized Peptide Nanofibers as Growth Stimulators for Regeneration of Peripheral Neurons. Advanced Functional Materials 2019, 0, 1809112.

12. Sieste, S.; Mack, T.; Synatschke, C. V.; Schilling, C.; Meyer Zu Reckendorf, C.; Pendi, L.; Harvey, S.; Ruggeri, F. S.; Knowles, T. P. J.; Meier, C.; Ng, D. Y. W.; Weil, T.; Knoll, B. Water-Dispersible Polydopamine-Coated Nanofibers for Stimulation of Neuronal Growth and Adhesion. Adv Healthc Mater 2018, 7, e1701485.

13. Müller, T.; Ruggeri, F. S.; Kulik, A. J.; Shimanovich, U.; Mason, T. O.; Knowles, T. P. J.; Dietler, G. Nanoscale spatially resolved infrared spectra from single microdroplets. Lab Chip 2014, 14, 1315-1319.

14. Shimanovich, U.; Ruggeri, F. S.; De Genst, E.; Adamcik, J.; Barros, T. P.; Porter, D.; Muller, T.; Mezzenga, R.; Dobson, C. M.; Vollrath, F.; Holland, C.; Knowles, T. P. J. Silk micrococoons for protein stabilisation and molecular encapsulation. Nat Commun 2017, 8, 15902.

15. Ruggeri, F. S.; Adamcik, J.; Jeong, J. S.; Lashuel, H. A.; Mezzenga, R.; Dietler, G. Influence of the beta-sheet content on the mechanical properties of aggregates during amyloid fibrillization. Angew Chem Int Ed Engl 2015, 54, 2462-6.

16. Ruggeri, F. S.; Habchi, J.; Cerreta, A.; Dietler, G. AFM-Based Single Molecule Techniques: Unraveling the Amyloid Pathogenic Species. Curr Pharm Des 2016, 22, 3950-70.

17. Tuttle, M. D.; Comellas, G.; Nieuwkoop, A. J.; Covell, D. J.; Berthold, D. A.; Kloepper, K. D.; Courtney, J. M.; Kim, J. K.; Barclay, A. M.; Kendall, A.; Wan, W.; Stubbs, G.; Schwieters, C. D.; Lee, V. M. Y.; George, J. M.; Rienstra, C. M. Solid-State NMR Structure of a Pathogenic Fibril of Full-Length Human $\alpha$-Synuclein. Nature structural \& molecular biology 2016, 23, 409-415.

18. Li, B.; Ge, P.; Murray, K. A.; Sheth, P.; Zhang, M.; Nair, G.; Sawaya, M. R.; Shin, W. S.; Boyer, D. R.; Ye, S.; Eisenberg, D. S.; Zhou, Z. H.; Jiang, L. Cryo-EM of full-length $\alpha$-synuclein reveals fibril polymorphs with a common structural kernel. Nature Communications 2018, 9, 3609. 
19. Guerrero-Ferreira, R.; Taylor, N. M. I.; Arteni, A.-A.; Kumari, P.; Mona, D.; Ringler, P.; Britschgi, M.; Lauer, M. E.; Makky, A.; Verasdonck, J.; Riek, R.; Melki, R.; Meier, B. H.; Böckmann, A.; Bousset, L.; Stahlberg, H. Two new polymorphic structures of human full-length alpha-synuclein fibrils solved by cryo-electron microscopy. eLife 2019, 8, e48907.

20. Adamcik, J.; Mezzenga, R. Amyloid Polymorphism in the Protein Folding and Aggregation Energy Landscape. Angewandte Chemie - International Edition 2018, 57, 8370-8382.

21. Flagmeier, P.; Meisl, G.; Vendruscolo, M.; Knowles, T. P.; Dobson, C. M.; Buell, A. K.; Galvagnion, C. Mutations associated with familial Parkinson's disease alter the initiation and amplification steps of alpha-synuclein aggregation. Proc Natl Acad Sci U S A 2016, 113, 1032833.

22. Buell, A. K.; Galvagnion, C.; Gaspar, R.; Sparr, E.; Vendruscolo, M.; Knowles, T. P. J.; Linse, S.; Dobson, C. M. Solution conditions determine the relative importance of nucleation and growth processes in \$a\$-synuclein aggregation. Proc. Natl. Acad. Sci. U. S. A. 2014, 111, 7671-7676.

23. Vácha, R.; Linse, S.; Lund, M. Surface Effects on Aggregation Kinetics of Amyloidogenic Peptides. Journal of the American Chemical Society 2014, 136, 11776-11782.

24. Peng, C.; Gathagan, R. J.; Covell, D. J.; Medellin, C.; Stieber, A.; Robinson, J. L.; Zhang, B.; Pitkin, R. M.; Olufemi, M. F.; Luk, K. C.; Trojanowski, J. Q.; Lee, V. M. Cellular milieu imparts distinct pathological alpha-synuclein strains in alpha-synucleinopathies. Nature 2018, 557, 558563.

25. Spillantini, M.; Schmidt, M.; Lee, V.; Trojanowski, J. $\alpha$-Synuclein in Lewy bodies. Nature 1997.

26. Lashuel, H. A.; Overk, C. R.; Oueslati, A.; Masliah, E. The many faces of $\alpha$-synuclein: from structure and toxicity to therapeutic target. Nature Reviews Neuroscience 2012, 14, 38-48.

27. Burré, J. The Synaptic Function of $\alpha$-Synuclein. J Parkinsons Dis 2015, 5, 699-713.

28. Kruger, R.; Kuhn, W.; Muller, T.; Woitalla, D.; Graeber, M.; Kosel, S.; Przuntek, H.; Epplen, J. T.; Schols, L.; Riess, O. Ala30Pro mutation in the gene encoding alpha-synuclein in Parkinson's disease. Nat Genet 1998, 18, 106-8.

29. Zarranz, J. J.; Alegre, J.; Gomez-Esteban, J. C.; Lezcano, E.; Ros, R.; Ampuero, I.; Vidal, L.; Hoenicka, J.; Rodriguez, O.; Atares, B.; Llorens, V.; Gomez Tortosa, E.; del Ser, T.; Munoz, D. G.; de Yebenes, J. G. The new mutation, E46K, of alpha-synuclein causes Parkinson and Lewy body dementia. Ann Neurol 2004, 55, 164-73.

30. Khalaf, O.; Fauvet, B.; Oueslati, A.; Dikiy, I.; Mahul-Mellier, A. L.; Ruggeri, F. S.; Mbefo, M. K.; Vercruysse, F.; Dietler, G.; Lee, S. J.; Eliezer, D.; Lashuel, H. A. The H50Q mutation enhances alpha-synuclein aggregation, secretion, and toxicity. The Journal of Biological Chemistry 2014, 289, 21856-76.

31. Lesage, S.; Anheim, M.; Letournel, F.; Bousset, L.; Honore, A.; Rozas, N.; Pieri, L.; Madiona, K.; Durr, A.; Melki, R.; Verny, C.; Brice, A. G51D alpha-synuclein mutation causes a novel parkinsonian-pyramidal syndrome. Ann Neurol 2013, 73, 459-71.

32. Polymeropoulos, M. H.; Lavedan, C.; Leroy, E.; Ide, S. E.; Dehejia, A.; Dutra, A.; Pike, B.; Root, H.; Rubenstein, J.; Boyer, R.; Stenroos, E. S.; Chandrasekharappa, S.; Athanassiadou, A.; Papapetropoulos, T.; Johnson, W. G.; Lazzarini, A. M.; Duvoisin, R. C.; Di Iorio, G.; Golbe, L. I.; Nussbaum, R. L. Mutation in the alpha-synuclein gene identified in families with Parkinson's disease. Science 1997, 276, 2045-7.

33. Ruggeri, F. S.; Šneideris, T.; Vendruscolo, M.; Knowles, T. P. J. Atomic force microscopy for single molecule characterisation of protein aggregation. Archives of Biochemistry and Biophysics 2019, 664, 134-148.

34. Ruggeri, F. S.; Charmet, J.; Kartanas, T.; Peter, Q.; Chia, S.; Habchi, J.; Dobson, C. M.; Vendruscolo, M.; Knowles, T. P. J. Microfluidic deposition for resolving single-molecule protein architecture and heterogeneity. Nature Communications 2018, 9, 3890.

35. De, S.; Whiten, D. R.; Ruggeri, F. S.; Hughes, C.; Rodrigues, M.; Sideris, D. I.; Taylor, C. G.; Aprile, F. A.; Muyldermans, S.; Knowles, T. P. J.; Vendruscolo, M.; Bryant, C.; Blennow, K.; Skoog, I.; Kern, S.; Zetterberg, H.; Klenerman, D. Soluble aggregates present in cerebrospinal fluid change in size and mechanism of toxicity during Alzheimer's disease progression. Acta Neuropathologica Communications 2019, 7, 120.

36. Chiki, A.; DeGuire, S. M.; Ruggeri, F. S.; Sanfelice, D.; Ansaloni, A.; Wang, Z.-M.; Cendrowska, U.; Burai, R.; Vieweg, S.; Pastore, A.; Dietler, G.; Lashuel, H. A. Mutant Exon1 Huntingtin 
Aggregation is Regulated by T3 Phosphorylation-Induced Structural Changes and Crosstalk between T3 Phosphorylation and Acetylation at K6. Angewandte Chemie 2017, 129, 5286-5291.

37. Adamcik, J.; Mezzenga, R. Study of amyloid fibrils via atomic force microscopy. Current Opinion in Colloid and Interface Science 2012, 17, 369-376.

38. Campioni, S.; Carret, G.; Jordens, S.; Nicoud, L.; Mezzenga, R.; Riek, R. The presence of an airwater interface affects formation and elongation of alpha-Synuclein fibrils. J Am Chem Soc 2014, $136,2866-75$.

39. Galvagnion, C.; Brown, J. W.; Ouberai, M. M.; Flagmeier, P.; Vendruscolo, M.; Buell, A. K.; Sparr, E.; Dobson, C. M. Chemical properties of lipids strongly affect the kinetics of the membrane-induced aggregation of alpha-synuclein. Proc Natl Acad Sci U S A 2016, 113, 7065-70.

40. Knowles, T. P.; Waudby, C. A.; Devlin, G. L.; Cohen, S. I.; Aguzzi, A.; Vendruscolo, M.; Terentjev, E. M.; Welland, M. E.; Dobson, C. M. An analytical solution to the kinetics of breakable filament assembly. Science 2009, 326, 1533-7.

41. Bolognesi, B.; Kumita, J. R.; Barros, T. P.; Esbjorner, E. K.; Luheshi, L. M.; Crowther, D. C.; Wilson, M. R.; Dobson, C. M.; Favrin, G.; Yerbury, J. J. ANS Binding Reveals Common Features of Cytotoxic Amyloid Species. ACS Chemical Biology 2010, 5, 735-740.

42. Bongiovanni, M. N.; Godet, J.; Horrocks, M. H.; Tosatto, L.; Carr, A. R.; Wirthensohn, D. C.; Ranasinghe, R. T.; Lee, J.-E.; Ponjavic, A.; Fritz, J. V.; Dobson, C. M.; Klenerman, D.; Lee, S. F. Multi-dimensional super-resolution imaging enables surface hydrophobicity mapping. Nature Communications 2016, 7, 13544.

43. Ruggeri, F. S.; Longo, G.; Faggiano, S.; Lipiec, E.; Pastore, A.; Dietler, G. Infrared nanospectroscopy characterization of oligomeric and fibrillar aggregates during amyloid formation. Nat Commun 2015, 6, 7831.

44. Barth, A. Infrared spectroscopy of proteins. Biochim Biophys Acta 2007, 1767, 1073-101.

45. Yang, H.; Yang, S.; Kong, J.; Dong, A.; Yu, S. Obtaining information about protein secondary structures in aqueous solution using Fourier transform IR spectroscopy. Nat Protoc 2015, 10, $382-$ 96.

46. Qamar, S.; Wang, G.; Randle, S. J.; Ruggeri, F. S.; Varela, J. A.; Lin, J. Q.; Phillips, E. C.; Miyashita, A.; Williams, D.; Strohl, F.; Meadows, W.; Ferry, R.; Dardov, V. J.; Tartaglia, G. G.; Farrer, L. A.; Kaminski Schierle, G. S.; Kaminski, C. F.; Holt, C. E.; Fraser, P. E.; Schmitt-Ulms, G.; Klenerman, D.; Knowles, T.; Vendruscolo, M.; St George-Hyslop, P. FUS Phase Separation Is Modulated by a Molecular Chaperone and Methylation of Arginine Cation-pi Interactions. Cell 2018, 173, 720-734 e15.

47. Theillet, F. X.; Binolfi, A.; Bekei, B.; Martorana, A.; Rose, H. M.; Stuiver, M.; Verzini, S.; Lorenz, D.; van Rossum, M.; Goldfarb, D.; Selenko, P. Structural disorder of monomeric alpha-synuclein persists in mammalian cells. Nature 2016, 530, 45-50.

48. Ruggeri, F. S.; Marcott, C.; Dinarelli, S.; Longo, G.; Girasole, M.; Dietler, G.; Knowles, T. P. J. Identification of Oxidative Stress in Red Blood Cells with Nanoscale Chemical Resolution by Infrared Nanospectroscopy. Int J Mol Sci 2018, 19, 2582.

49. Debelouchina, G. T.; Bayro, M. J.; Fitzpatrick, A. W.; Ladizhansky, V.; Colvin, M. T.; Caporini, M. A.; Jaroniec, C. P.; Bajaj, V. S.; Rosay, M.; MacPhee, C. E.; Vendruscolo, M.; Maas, W. E.; Dobson, C. M.; Griffin, R. G. Higher Order Amyloid Fibril Structure by MAS NMR and DNP Spectroscopy. Journal of the American Chemical Society 2013, 135, 19237-19247.

50. Ruggeri, F. S.; Vieweg, S.; Cendrowska, U.; Longo, G.; Chiki, A.; Lashuel, H. A.; Dietler, G. Nanoscale studies link amyloid maturity with polyglutamine diseases onset. Sci Rep 2016, 6, 31155 .

51. Usov, I.; Mezzenga, R. Correlation between Nanomechanics and Polymorphic Conformations in Amyloid Fibrils. Acs Nano 2014, 8, 11035-11041.

52. Knowles, T. P.; Buehler, M. J. Nanomechanics of functional and pathological amyloid materials. Nat Nanotechnol 2011, 6, 469-79.

53. Adamcik, J.; Jung, J. M.; Flakowski, J.; De Los Rios, P.; Dietler, G.; Mezzenga, R. Understanding amyloid aggregation by statistical analysis of atomic force microscopy images. Nat. Nanotechnol. 2010, 5, 423.

54. Knowles, T. P.; De Simone, A.; Fitzpatrick, A. W.; Baldwin, A.; Meehan, S.; Rajah, L.; Vendruscolo, M.; Welland, M. E.; Dobson, C. M.; Terentjev, E. M. Twisting transition between crystalline and fibrillar phases of aggregated peptides. Phys Rev Lett 2012, 109, 158101. 
55. Pashuck, E. T.; Stupp, S. I. Direct Observation of Morphological Tranformation from Twisted Ribbons into Helical Ribbons. Journal of the American Chemical Society 2010, 132, 8819-8821.

56. Reynolds, N. P.; Adamcik, J.; Berryman, J. T.; Handschin, S.; Zanjani, A. A. H.; Li, W.; Liu, K.; Zhang, A.; Mezzenga, R. Competition between crystal and fibril formation in molecular mutations of amyloidogenic peptides. Nat Commun 2017, 8, 1338.

57. Ionescu-Zanetti, C.; Khurana, R.; Gillespie, J. R.; Petrick, J. S.; Trabachino, L. C.; Minert, L. J.; Carter, S. A.; Fink, A. L. Monitoring the assembly of Ig light-chain amyloid fibrils by atomic force microscopy. Proceedings of the National Academy of Sciences 1999, 96, 13175-13179.

58. Chan, P. K.; Chattopadhyay, M.; Sharma, S.; Souda, P.; Gralla, E. B.; Borchelt, D. R.; Whitelegge, J. P.; Valentine, J. S. Structural similarity of wild-type and ALS-mutant superoxide dismutase-1 fibrils using limited proteolysis and atomic force microscopy. Proceedings of the National Academy of Sciences 2013, 110, 10934-10939.

59. Kad, N. M.; Myers, S. L.; Smith, D. P.; Alastair Smith, D.; Radford, S. E.; Thomson, N. H. Hierarchical Assembly of $\beta 2$-Microglobulin Amyloid In Vitro Revealed by Atomic Force Microscopy. Journal of Molecular Biology 2003, 330, 785-797.

60. Khurana, R.; Ionescu-Zanetti, C.; Pope, M.; Li, J.; Nielson, L.; Ramírez-Alvarado, M.; Regan, L.; Fink, A. L.; Carter, S. A. A General Model for Amyloid Fibril Assembly Based on Morphological Studies Using Atomic Force Microscopy. Biophysical Journal 2003, 85, 1135-1144.

61. Knowles, T. P.; Fitzpatrick, A. W.; Meehan, S.; Mott, H. R.; Vendruscolo, M.; Dobson, C. M.; Welland, M. E. Role of Intermolecular Forces in Defining Material Properties of Protein Nanofibrils. Science 2007, 318, 1900-1903.

62. Fares, M. B.; Ait-Bouziad, N.; Dikiy, I.; Mbefo, M. K.; Jovicic, A.; Kiely, A.; Holton, J. L.; Lee, S. J.; Gitler, A. D.; Eliezer, D.; Lashuel, H. A. The novel Parkinson's disease linked mutation G51D attenuates in vitro aggregation and membrane binding of alpha-synuclein, and enhances its secretion and nuclear localization in cells. Hum Mol Genet 2014, 23, 4491-509.

63. Conway, K. A.; Lee, S.-J.; Rochet, J.-C.; Ding, T. T.; Williamson, R. E.; Lansbury, P. T. Acceleration of oligomerization, not fibrillization, is a shared property of both alpha -synuclein mutations linked to early-onset Parkinson's disease: Implications for pathogenesis and therapy. Proceedings of the National Academy of Sciences 2000, 97, 571-576.

64. Narhi, L.; Wood, S. J.; Steavenson, S.; Jiang, Y.; Wu, G. M.; Anafi, D.; Kaufman, S. A.; Martin, F.; Sitney, K.; Denis, P.; Louis, J. C.; Wypych, J.; Biere, A. L.; Citron, M. Both familial Parkinson's disease mutations accelerate alpha-synuclein aggregation. The Journal of biological chemistry 1999, 274, 9843-6.

65. Comellas, G.; Lemkau, L. R.; Zhou, D. H.; George, J. M.; Rienstra, C. M. Structural Intermediates during $\alpha$-Synuclein Fibrillogenesis on Phospholipid Vesicles. Journal of the American Chemical Society 2012, 134, 5090-5099.

66. Lemkau, L. R.; Comellas, G.; Kloepper, K. D.; Woods, W. S.; George, J. M.; Rienstra, C. M. Mutant protein A30P alpha-synuclein adopts wild-type fibril structure, despite slower fibrillation kinetics. J Biol Chem 2012, 287, 11526-32.

67. Klein, C.; Westenberger, A. Genetics of Parkinson's disease. Cold Spring Harb Perspect Med 2012, 2, a008888-a008888.

68. Papapetropoulos, S.; Adi, N.; Ellul, J.; Argyriou, A. A.; Chroni, E. A prospective study of familial versus sporadic Parkinson's disease. Neurodegener Dis 2007, 4, 424-7.

69. Qiang, W.; Yau, W. M.; Lu, J. X.; Collinge, J.; Tycko, R. Structural variation in amyloid-beta fibrils from Alzheimer's disease clinical subtypes. Nature 2017, 541, 217-221.

70. Li, Y.; Zhao, C.; Luo, F.; Liu, Z.; Gui, X.; Luo, Z.; Zhang, X.; Li, D.; Liu, C.; Li, X. Amyloid fibril structure of $\alpha$-synuclein determined by cryo-electron microscopy. Cell Research 2018, 28, 897903. 